\title{
CONSISTENT STRUCTURES OF INVARIANT QUADRATURE RULES FOR THE $n$-SIMPLEX
}

\author{
J. I. MAEZTU AND E. SAINZ DE LA MAZA
}

\begin{abstract}
In this paper we develop a technique to obtain, in a systematic way, the consistency conditions for the $n$-dimensional simplex $T_{n}$ for any dimension $n$ and degree of precision $d$. The introduction of a convenient basis of invariant polynomials provides a powerful tool to analyze and obtain consistent structures. We also present tables listing the optimal consistent structures for dimensions $n=2, \ldots, 8$ and degree of precision up to $d=23$. This paper is devoted only to structures. No quadrature rules are presented here.
\end{abstract}

\section{INTRODUCTION}

Constructing quadrature formulas with a given structure requires the solution of a system of moment equations. These are nonlinear and the system can be very large. Such a major computational task may be helped if information about structures for which there is likely to be a solution is available. Some information of this type is provided by a set of consistency conditions. We develop here a theory which allows one to obtain the set of consistency conditions for the system of nonlinear equations arising for the $n$-simplex. For example, this would allow a solver to discard a priori many structures (the nonconsistent ones) whose corresponding systems of moment equations do not have any solution.

Let $T_{n}$ denote a nondegenerate simplex in the $n$-dimensional Euclidean space $\mathbb{R}^{n}$ and let $\mathscr{G}_{n}$ be the symmetry group of $T_{n}$, that is, the set of all affine transformations $\mathbf{s}: \mathbb{R}^{n} \rightarrow \mathbb{R}^{n}$ that leave $T_{n}$ invariant. For every $\mathbf{x} \in \mathbb{R}^{n}$, let $\nu(\mathbf{x})$ denote the number of different points in the so-called orbit of $\mathbf{x}$, that is,

$$
\nu(\mathbf{x})=\operatorname{card}\left\{\mathbf{s}(\mathbf{x}): \mathbf{s} \in \mathscr{G}_{n}\right\} .
$$

The value $\nu(\mathbf{x})$ depends on the relative position of the point $\mathbf{x}$ in the simplex $T_{n}$. More specifically, $\nu(\mathbf{x})$ is determined by the form taken by the barycentric representation of $\mathbf{x}$ (see later).

Let $\mathbb{N}$ denote the set of all nonnegative integers. Given a point $\mathbf{x}=\left(x_{1}, \ldots\right.$, $\left.x_{n}\right) \in \mathbb{R}^{n}$ and a multi-index $\boldsymbol{\alpha}=\left(\alpha_{1}, \ldots, \alpha_{n}\right) \in \mathbb{N}^{n}$, we denote as usual $\mathbf{x}^{\boldsymbol{\alpha}}=$

Received by the editor June 2, 1993 and, in revised form, February 21, 1994, June 2, 1994, and July 5, 1994.

1991 Mathematics Subject Classification. Primary 65D32, 65D30.

Key words and phrases. Invariant quadrature rule, consistency conditions, simplex, consistent structure, multidimensional quadrature.

(C)1995 American Mathematical Society 
$x_{1}{ }^{\alpha_{1}} \cdots x_{n}{ }^{\alpha_{n}}$ and $|\boldsymbol{\alpha}|=\alpha_{1}+\cdots+\alpha_{n}$. Let $\mathscr{P}$ be the space of real polynomials in the $n$ variables $x_{1}, \ldots, x_{n}$. Let $\mathscr{P}_{d}$ denote the space of real polynomials of degree not greater than $d$ and let $\mathscr{H}_{d}$ denote the space of real and homogeneous polynomials of degree $d$. That is, $\mathscr{P}_{d}=\operatorname{span}\left\{\mathbf{x}^{\alpha}: \alpha \in \mathbb{N}^{n},|\boldsymbol{\alpha}| \leq d\right\}$ and $\mathscr{H}_{d}=$ $\operatorname{span}\left\{\mathbf{x}^{\boldsymbol{\alpha}}: \boldsymbol{\alpha} \in \mathbb{N}^{n},|\boldsymbol{\alpha}|=d\right\}$.

Definition 1. A polynomial $p \in \mathscr{P}$ is said to be invariant when

$$
p(\mathbf{s}(\mathbf{x}))=p(\mathbf{x}) \quad \forall \mathbf{s} \in \mathscr{G}_{n}, \quad \forall \mathbf{x} \in \mathbb{R}^{n}
$$

Let us denote by $\mathscr{P}^{*}$ the space of all invariant polynomials of $\mathscr{P}$, and let $\mathscr{P}_{d}^{*}=\mathscr{P}^{*} \cap \mathscr{P}_{d}$ and $\mathscr{H}_{d}^{*}=\mathscr{P}^{*} \cap \mathscr{H}_{d}$ be the spaces of all invariant polynomials of $\mathscr{P}_{d}$ and $\mathscr{H}_{d}$, respectively.

Now, let us consider the integral

$$
I(f)=\frac{1}{\operatorname{vol}\left(T_{n}\right)} \int_{T_{n}} f(\mathbf{x}) d \mathbf{x}
$$

and the problem of constructing a quadrature rule

$$
Q(f)=\sum_{i=1}^{\nu(Q)} W_{i} f\left(\mathbf{x}_{i}\right)
$$

that approximates $I(f)$ with a given degree of precision $d$, that is, verifying

$$
Q(p)=I(p) \quad \forall p \in \mathscr{P}_{d},
$$

and having a number of nodes, say $\nu(Q)$, as low as possible. A familiar approach to this problem is by considering the coordinates of nodes $\mathbf{x}_{i}$ and the weights $W_{i}$ of the rule as unknown parameters that will be obtained by fitting the moment equations

$$
Q\left(\mathbf{x}^{\alpha}\right)-I\left(\mathbf{x}^{\alpha}\right)=0, \quad|\boldsymbol{\alpha}| \leq d .
$$

For every $\mathbf{s} \in \mathscr{G}_{n}$, we have $\mathbf{s}\left(T_{n}\right)=T_{n}$ and hence, $I(f \circ \mathbf{s})=I(f)$. This means that integration over $T_{n}$ is an axis-independent concept in the sense described in Bez [2]. Therefore it is convenient to use for computing $I(f)$ an algorithm $Q(f)$ with the same independence property, according to the definition below.

Definition 2. A quadrature rule (1.4) is said to be invariant when it satisfies

$$
Q(f \circ \mathbf{s})=Q(f) \quad \forall \mathbf{s} \in \mathscr{G}_{n} .
$$

An invariant quadrature rule can be written in the form (see [14])

$$
Q(f)=\sum_{i=1}^{\eta} W_{i} \nu\left(\mathbf{x}_{i}\right) R\left(f ; \mathbf{x}_{i}\right),
$$

where the numbers $\nu\left(\mathbf{x}_{i}\right)$ are defined by (1.1) and the so-called basic rules $R\left(f ; \mathbf{x}_{i}\right)$ are given by

$$
R\left(f ; \mathbf{x}_{i}\right)=\frac{1}{(n+1) !} \sum_{\mathbf{s} \in \mathscr{G}_{n}} f\left(\mathbf{s}\left(\mathbf{x}_{i}\right)\right) .
$$


Let us note that the number of separate function values, i.e., the number of nodes, involved in a basic rule (1.9) is $\nu\left(\mathbf{x}_{i}\right)$. In fact, $R\left(f ; \mathbf{x}_{i}\right)$ can be written as

$$
R\left(f ; \mathbf{x}_{i}\right)=\frac{1}{\nu\left(\mathbf{x}_{i}\right)} \sum_{\mathbf{z} \in \operatorname{orb}\left(\mathbf{x}_{i}\right)} f(\mathbf{z}) .
$$

As in [14], we use this characteristic to classify basic rules in different types, where the form that takes the barycentric representation of the generator node $\mathbf{x}_{i}$ is what determines the type of the corresponding basic rule $R\left(f ; \mathbf{x}_{i}\right)$. Then, the so-called rule structure of an invariant quadrature rule (1.8) is given in the form

$$
\left(K_{0}, K_{1}, \ldots, K_{M_{n}}\right) \text {, }
$$

where the numbers $K_{i}$ indicate how many basic rules of each type are involved in (1.8).

For a given rule structure, the generic form of the corresponding invariant quadrature rule contains a number of unknown parameters that is considerably smaller than in the case of a generic noninvariant quadrature rule with a similar number of nodes. But, on the other hand, this fact is partially compensated by a substantial reduction on the number of moment equations to be satisfied, as can be deduced from the following well-known theorem.

Theorem 1 (Sobolev). An invariant quadrature rule (1.8) has degree of precision $d$ if and only if

$$
Q(p)=I(p) \quad \forall p \in \mathscr{P}_{d}^{*} .
$$

The search for rule structures satisfying certain consistency conditions is a first step towards the construction of a quadrature rule of a given degree of precision. The rule structure is essential not only to determine the (expected) number of nodes of the rule, but also because it defines the functional dependence on the unknowns in the (reduced) system of moment equations.

These consistency conditions are linear inequalities to be satisfied by a rule structure (1.11) in order to guarantee that, if the quadrature problem is considered as solving a system of nonlinear equations, then each subsystem will have a number of unknowns larger than or equal to the number of equations. They attempt to ensure that a quadrature rule can be of a given degree of precision $d$, but for the nonlinear system of moment equations the hazards of nonlinear relationships and complex solutions still remain. Thus, strictly mathematically, consistency conditions are not necessary nor sufficient for the existence of solutions of a quadrature problem.

There have been many authors who have contributed to the calculation of quadrature rules for multidimensional regions. Basic references on this field are $[4,9,14$ and 20]. More specific results for 2-dimensional polygons are given in $[3,5,7,15$ and 16$]$. Some results for the $n$-dimensional simplex are given in [1, 10 and 12]. The introduction of some kind of consistency conditions is mainly due to Keast, Lyness, Mantel, Rabinowitz and Richter (see [18] and [19] for 2and 3-dimensional regions, and [14] for more general cases). These conditions are difficult to apply in high dimensions, owing to the lack of closed expressions to calculate the dimensions of the null spaces of invariant polynomials. In this 
paper we give a systematic approach to the consistency conditions that hold for a general $n$-dimensional simplex, and develop a technique to obtain, at least computationally, the dimensions of the null spaces of invariant polynomials.

We now give a brief description of the sections of this paper. In $\S 2$ we describe the structure of an invariant quadrature rule for the $n$-dimensional simplex $T_{n}$, and in $\S 3$ we analyze and calculate the dimensions of the spaces of invariant polynomials and give recurrence relations to obtain them. We also define a convenient basis of invariant polynomials, which simplifies greatly the calculation of these dimensions. In $\S 4$ we develop the theory to a point where we can define the consistency conditions of an invariant quadrature rule. In $\S 5$ we give some details of how these structures are obtained in practice and of how some of the various computational problems encountered are treated. We finish with listings of the optimal structures for dimensions $n=2$ up to $n=8$.

\section{STRUCTURE OF AN INVARIANT QUADRATURE RULE}

Barycentric coordinates are a useful tool to study questions related with the symmetry group of a simplex $T_{n}$. Let $\mathbf{v}_{0}, \ldots, \mathbf{v}_{n}$ denote the $n+1$ vertices of $T_{n}$. The barycentric coordinates $\lambda=\lambda(\mathbf{x})$ of a point $\mathbf{x} \in \mathbb{R}^{n}$ are defined by

$$
\lambda=\left(\lambda_{0}, \ldots, \lambda_{n}\right): \sum_{j=0}^{n} \lambda_{j}=1, \quad \sum_{j=0}^{n} \lambda_{j} \mathbf{v}_{j}=\mathbf{x} .
$$

It is well known that each symmetry $\mathbf{s} \in \mathscr{G}_{n}$ can be identified with a permutation of the barycentric coordinates, that is,

$$
\lambda_{j}(\mathbf{s}(\mathbf{x}))=\lambda_{\pi_{j}}(\mathbf{x}), j=0, \ldots, n,
$$

where $\left(\pi_{0}, \ldots, \pi_{n}\right)$ is a permutation of $(0, \ldots, n)$. Therefore, $\mathscr{G}_{n}$ consist of $(n+1)$ ! symmetries and, for each $\mathbf{x} \in \mathbb{R}^{n}$, the number $\nu(\mathbf{x})$ defined in $(1.1)$ is the same as the number of different points obtained permuting the barycentric coordinates of $\mathbf{x}$. This suggests a classification of the points in $\mathbb{R}^{n}$ according to the following

Definition 3. For a given $n$ let $r$ and $m_{0}, \ldots, m_{r}$ be positive integers satisfying

$$
m_{0} \geq m_{1} \geq \cdots \geq m_{r} \geq 1, \quad \sum_{j=0}^{r} m_{j}=n+1 .
$$

A class $\left[m_{0}, m_{1}, \ldots, m_{r}\right]$ is defined as the set of points $\mathbf{x} \in \mathbb{R}^{n}$ such that for some symmetry $\mathbf{s} \in \mathscr{G}_{n}$ the barycentric coordinates representation of $\mathbf{s}(\mathbf{x})$ has the form

$$
\lambda(\mathbf{s}(\mathbf{x}))=(\overbrace{a_{0}, \ldots, a_{0}}^{m_{0} \text { times }}, \overbrace{a_{1}, \ldots, a_{1}}^{m_{1} \text { times }}, \ldots, \overbrace{a_{r}, \ldots, a_{r}}^{m_{r} \text { times }}),
$$

with

$$
\sum_{j=0}^{r} m_{j} a_{j}=1
$$

From a geometric point of view the class $\left[m_{0}, m_{1}, \ldots, m_{r}\right]$ coincides with the set $\left\{\mathbf{s}(V): \mathbf{s} \in \mathscr{G}_{n}\right\}$, where $V$ denotes the $r$-dimensional affine manifold 
TABLE 1. Classes and types of basic rules for dimensions $n=2$ to 7

\begin{tabular}{|c|c|c|c|c|c|c|c|c|c|}
\hline$n$ & & Class (Type) & $r_{i}+1 \mid$ & $\nu_{i}$ & & & & & \\
\hline 2 & $\mathscr{C}_{0}$ & $=[3]$ & 1 & 1 & $n$ & & Class (Type) & $r_{i}+1$ & $\nu_{i}$ \\
\hline 2 & $\mathscr{C}_{1}$ & $=[2,1]$ & 2 & 3 & 3 & $\mathscr{C}_{0}$ & $=[4]$ & 1 & 1 \\
\hline 2 & $\mathscr{C}_{2}$ & $=[1,1,1]$ & 3 & 6 & 3 & $\mathscr{C}_{1}$ & $=[3,1]$ & 2 & 4 \\
\hline 4 & $\mathscr{C}_{0}$ & $=[5]$ & 1 & 1 & 3 & $\mathscr{C}_{2}$ & $=[2,2]$ & 2 & 6 \\
\hline 4 & $\mathscr{C}_{1}$ & $=[4,1]$ & 2 & 5 & 3 & $\mathscr{C}_{3}$ & $=[2,1,1]$ & 3 & 12 \\
\hline 4 & $\mathscr{C}_{2}$ & $=[3,2]$ & 2 & 10 & 3 & $\mathscr{C}_{4}$ & $=[1,1,1,1]$ & 4 & 24 \\
\hline 4 & $\mathscr{C}_{3}$ & $=[3,1,1]$ & 3 & 20 & 5 & $\mathscr{C}_{0}$ & $=[6]$ & 1 & 1 \\
\hline 4 & $\mathscr{C}_{4}$ & $=[2,2,1]$ & 3 & 30 & 5 & $\mathscr{C}_{1}$ & $=[5,1]$ & 2 & 6 \\
\hline 4 & $\mathscr{C}_{5}$ & $=[2,1,1,1]$ & 4 & 60 & 5 & $\mathscr{C}_{2}$ & $=[4,2]$ & 2 & 15 \\
\hline 4 & $\mathscr{C}_{6}$ & $=[1,1,1,1,1]$ & 5 & 120 & 5 & $\mathscr{C}_{3}$ & $=[3,3]$ & 2 & 20 \\
\hline 7 & $\mathscr{C}_{0}$ & $=[8]$ & 1 & 1 & 5 & $\mathscr{C}_{4}$ & $=[4,1,1]$ & 3 & 30 \\
\hline 7 & $\mathscr{C}_{1}$ & $=[7,1]$ & 2 & 8 & 5 & $\mathscr{C}_{5}$ & $=[3,2,1]$ & 3 & 60 \\
\hline 7 & $\mathscr{C}_{2}$ & $=[6,2]$ & 2 & 28 & 5 & $\mathscr{C}_{6}$ & $=[2,2,2]$ & 3 & 90 \\
\hline 7 & $\mathscr{C}_{3}$ & $=[5,3]$ & 2 & 56 & 5 & $\mathscr{C}_{7}$ & $=[3,1,1,1]$ & 4 & 120 \\
\hline 7 & $\mathscr{C}_{4}$ & $=[4,4]$ & 2 & 70 & 5 & $\mathscr{C}_{8}$ & $=[2,2,1,1]$ & 4 & 180 \\
\hline 7 & $\mathscr{C}_{5}$ & $=[6,1,1]$ & 3 & 56 & 5 & $\mathscr{C}_{9}$ & $=[2,1,1,1,1]$ & 5 & 360 \\
\hline 7 & $\mathscr{C}_{6}$ & $=[5,2,1]$ & 3 & 168 & 5 & $\mathscr{C}_{10}$ & $=[1,1,1,1,1,1]$ & 6 & 720 \\
\hline 7 & $\mathscr{C}_{7}$ & $=[4,3,1]$ & 3 & 280 & 6 & $\mathscr{C}_{0}$ & $=[7]$ & 1 & 1 \\
\hline 7 & $\mathscr{C}_{8}$ & $=[4,2,2]$ & 3 & 420 & 6 & $\mathscr{C}_{1}$ & $=[6,1]$ & 2 & 7 \\
\hline $7 \xi$ & $\mathscr{C}_{9}$ & $=[3,3,2]$ & 3 & 560 & 6 & $\mathscr{C}_{2}$ & $=[5,2]$ & 2 & 21 \\
\hline 7 & $\mathscr{C}_{10}$ & $=[5,1,1,1]$ & 4 & 336 & 6 & $\mathscr{C}_{3}$ & $=[4,3]$ & 2 & 35 \\
\hline 7 & $\mathscr{C}_{11}$ & $=[4,2,1,1]$ & 4 & 840 & 6 & $\mathscr{C}_{4}$ & $=[5,1,1]$ & 3 & 42 \\
\hline $7 \xi$ & $\mathscr{E}_{12}$ & $=[3,3,1,1]$ & 4 & 1120 & 6 & $\mathscr{C}_{5}$ & $=[4,2,1]$ & 3 & 105 \\
\hline 7 & $\mathscr{C}_{13}$ & $=[3,2,2,1]$ & 4 & 1680 & 6 & $\mathscr{C}_{6}$ & $=[3,3,1]$ & 3 & 140 \\
\hline 7 & $\mathscr{C}_{14}$ & $=[2,2,2,2]$ & 4 & 2520 & 6 & $\mathscr{C}_{7}$ & $=[3,2,2]$ & 3 & 210 \\
\hline 7 & $\mathscr{C}_{15}$ & $=[4,1,1,1,1]$ & 5 & 1680 & 6 & $\mathscr{C}_{8}$ & $=[4,1,1,1]$ & 4 & 210 \\
\hline 7 & $\mathscr{C}_{16}$ & $=[3,2,1,1,1]$ & 5 & 3360 & 6 & $\mathscr{C}_{9}$ & $=[3,2,1,1]$ & 4 & 420 \\
\hline 7 & $\mathscr{C}_{17}$ & $=[2,2,2,1,1]$ & 5 & 5040 & 6 & $\mathscr{C}_{10}$ & $=[2,2,2,1]$ & 4 & 630 \\
\hline 7 & $\mathscr{C}_{18}$ & $=[3,1,1,1,1,1]$ & 6 & 6720 & 6 & $\mathscr{C}_{11}$ & $=[3,1,1,1,1]$ & 5 & 840 \\
\hline 7 & $\mathscr{C}_{19}$ & $=[2,2,1,1,1,1]$ & & 10080 & 6 & $\mathscr{C}_{12}$ & $=[2,2,1,1,1]$ & & 1260 \\
\hline 7 & $\mathscr{C}_{20}$ & $=[2,1,1,1,1,1,1]$ & & 20160 & 6 & $\mathscr{C}_{13}$ & $=[2,1,1,1,1,1]$ & & 2520 \\
\hline 7 & $\mathscr{C}_{21}$ & $=[1,1,1,1,1,1,1,1]$ & & 40320 & 6 & $\mathscr{C}_{14}$ & $=[1,1,1,1,1,1,1]$ & & 5040 \\
\hline
\end{tabular}

with parametric representation in barycentric coordinates given by the righthand side of (2.4) and by (2.5). Let us note that for a given $n$, the intersection of two classes is a class, and that all the classes contain the class $[n+1]$. 
TABLE 2. Classes and types of basic rules for dimension $n=8$

\begin{tabular}{|l|l|r|r|}
\hline$n$ & Class (Type) & $r_{i}+1$ & \multicolumn{1}{|c|}{$\nu_{i}$} \\
\hline 8 & $\mathscr{C}_{0}=[9]$ & 1 & 1 \\
8 & $\mathscr{C}_{1}=[8,1]$ & 2 & 9 \\
8 & $\mathscr{C}_{2}=[7,2]$ & 2 & 36 \\
8 & $\mathscr{C}_{3}=[6,3]$ & 2 & 84 \\
8 & $\mathscr{C}_{4}=[5,4]$ & 2 & 126 \\
8 & $\mathscr{C}_{5}=[7,1,1]$ & 3 & 72 \\
8 & $\mathscr{C}_{6}=[6,2,1]$ & 3 & 252 \\
8 & $\mathscr{C}_{7}=[5,3,1]$ & 3 & 504 \\
8 & $\mathscr{C}_{8}=[4,4,1]$ & 3 & 630 \\
8 & $\mathscr{C}_{9}=[5,2,2]$ & 3 & 756 \\
8 & $\mathscr{C}_{10}=[4,3,2]$ & 3 & 1260 \\
8 & $\mathscr{C}_{11}=[3,3,3]$ & 3 & 1680 \\
8 & $\mathscr{C}_{12}=[6,1,1,1]$ & 4 & 504 \\
8 & $\mathscr{C}_{13}=[5,2,1,1]$ & 4 & 1512 \\
8 & $\mathscr{C}_{14}=[4,3,1,1]$ & 4 & 2520 \\
\hline
\end{tabular}

\begin{tabular}{|l|l|r|r|}
\hline$n$ & \multicolumn{1}{|c|}{ Class (Type) } & $r_{i}+1$ & \multicolumn{1}{c|}{$\nu_{i}$} \\
\hline 8 & $\mathscr{C}_{15}=[4,2,2,1]$ & 4 & 3780 \\
8 & $\mathscr{C}_{16}=[3,3,2,1]$ & 4 & 5040 \\
8 & $\mathscr{C}_{17}=[3,2,2,2]$ & 4 & 7560 \\
8 & $\mathscr{C}_{18}=[5,1,1,1,1]$ & 5 & 3024 \\
8 & $\mathscr{C}_{19}=[4,2,1,1,1]$ & 5 & 7560 \\
8 & $\mathscr{C}_{20}=[3,3,1,1,1]$ & 5 & 10080 \\
8 & $\mathscr{C}_{21}=[3,2,2,1,1]$ & 5 & 15120 \\
8 & $\mathscr{C}_{22}=[2,2,2,2,1]$ & 5 & 22680 \\
8 & $\mathscr{C}_{23}=[4,1,1,1,1,1]$ & 6 & 15120 \\
8 & $\mathscr{C}_{24}=[3,2,1,1,1,1]$ & 6 & 30240 \\
8 & $\mathscr{C}_{25}=[2,2,2,1,1,1]$ & 6 & 45360 \\
8 & $\mathscr{C}_{26}=[3,1,1,1,1,1,1]$ & 7 & 60480 \\
8 & $\mathscr{C}_{27}=[2,2,1,1,1,1,1]$ & 7 & 90720 \\
8 & $\mathscr{C}_{28}=[2,1,1,1,1,1,1,1]$ & 8 & 181440 \\
8 & $\mathscr{C}_{29}=[1,1,1,1,1,1,1,1,1]$ & 9 & 362880 \\
\hline
\end{tabular}

For a given $n$, the inclusion relation between sets establishes a partial order relation, say $\prec$, among all the classes of points of $\mathbb{R}^{n}$. The corresponding relationship trees can be easily constructed using a concept similar to the contracted version given in [14]. For example, the set of integers $[3,1,1]$ may be obtained from the set $[2,1,1,1]$ by replacing 2,1 by $2+1$. Hence, we have $[3,1,1] \prec[2,1,1,1]$. In a similar way, the set $[2,2,1]$ may be obtained from the set $[2,1,1,1]$ by replacing 1,1 by $1+1$. So we also have $[2,2,1] \prec[2,1,1,1]$.

In Tables 1 and 2 we describe the different classes that are used in this paper. For each dimension $n$, the classes have been enumerated and are represented in the form

$$
\mathscr{C}_{i}=\left[m_{0}, m_{1}, \ldots, m_{r_{1}}\right], \quad i=0, \ldots, M_{n} .
$$

Let us note that the classes have been numbered in such a way that

$$
\mathscr{C}_{i} \prec \mathscr{C}_{j} \Longrightarrow i<j
$$

and therefore for every $n$ we have that $\mathscr{C}_{0}=[n+1]$ and $\mathscr{C}_{M_{n}}=[\overbrace{1, \ldots, 1}^{n+1 \text { times }}]$.

Definition 4. A basic rule $R\left(f ; \mathbf{x}_{i j}\right)$ is said to be of type $\mathscr{C}_{i}$ if and only if $\mathscr{C}_{i}$ is the smallest (i.e., the intersection) of all the classes to which the generator node $\mathbf{x}_{i j}$ belongs.

If $\mathscr{C}_{i}=\left[m_{0}, m_{1}, \ldots, m_{r_{1}}\right]$, the generic form of the nodes of a basic rule of type $\mathscr{C}_{i}$ is given by $(2.4)$, where $r=r_{i}$ and the parameters $a_{0}, a_{1}, \ldots, a_{r_{i}}$ are different and related by $(2.5)$. Hence, for each basic rule $R\left(f ; \mathbf{x}_{i j}\right)$ of type $\mathscr{C}_{i}$ we have $r_{i}$ free parameters and a number of nodes, say $\nu_{i}$, given by

$$
\nu_{i}=\nu\left(\mathbf{x}_{i j}\right)=\frac{(n+1) !}{m_{0} ! m_{1} ! \cdots m_{r_{1}} !} .
$$


Definition 5. For a given $n$, let $K_{0}, K_{1}, \ldots, K_{M_{n}}$ be nonnegative integers with $K_{0} \leq 1$. We say that an $n$-dimensional invariant quadrature rule (1.8) has a rule structure $\left(K_{0}, K_{1}, \ldots, K_{M_{n}}\right)$ when it can be written in the form

$$
Q(f)=\sum_{i=0}^{M_{n}} \sum_{j=1}^{K_{i}} W_{i j} \nu_{i} R\left(f ; \mathbf{x}_{i j}\right),
$$

where, for every $i=0, \ldots, M_{n},\left\{R\left(f ; \mathbf{x}_{i j}\right) ; j=1, \ldots, K_{i}\right\}$ is a set of $K_{i}$ different basic rules of type $\mathscr{C}_{i}$.

$K_{i}=0$ means that there is no basic rule of type $\mathscr{C}_{i}$ in (2.9). The only basic rule of type $\mathscr{C}_{0}$ is the one generated by the barycenter of the simplex. Therefore, $K_{0}$ can only take the values 0 or 1 .

Now, let us consider the problem of constructing an invariant quadrature rule with a given rule structure and a given degree of precision. The rule structure allows us to define, by using Tables 1 and 2, the generic (parametric) form of the rule. The global number of nodes of an invariant quadrature rule $Q$ with rule structure $\left(K_{0}, K_{1}, \ldots, K_{M_{n}}\right)$ is given by

$$
\nu(Q)=\sum_{i=0}^{M_{n}} \nu_{i} K_{i},
$$

and the number of free parameters to be determined by moment fitting is given by

$$
N_{P}(Q)=\sum_{i=0}^{M_{n}}\left(r_{i}+1\right) K_{i} .
$$

\section{ON THE SPACE OF INVARIANT POLYNOMIALS}

Sobolev's result (Theorem 1) shows that the degree of precision of an invariant quadrature rule only depends on its exactness for invariant polynomials. On the other hand, it is important for our purposes to analyze the space $\mathscr{P}^{*}$ of invariant polynomials in such a way that each of the spaces $\mathscr{H}_{d}^{*}$ of homogeneous and invariant polynomials of degree $d$ can be identified. This will be done by constructing a basis of $\mathscr{P}^{*}$ formed by homogeneous polynomials. Then we will have

$$
\mathscr{P}_{d}^{*}=\mathscr{H}_{0}^{*} \oplus \mathscr{H}_{1}^{*} \oplus \cdots \oplus \mathscr{H}_{d}^{*},
$$

and the dimension of the problems involved in the construction of consistent structures of invariant quadrature rules will be considerably reduced.

First of all, let us note that it is not difficult to obtain a basis of $\mathscr{P}_{d}$ by using the barycentric polynomials $\lambda_{j}=\lambda_{j}(\mathbf{x})$. Let $\boldsymbol{\beta}=\left(\beta_{0}, \beta_{1}, \ldots, \beta_{n}\right)$ denote a multi-index, $\boldsymbol{\beta} \in \mathbb{N}^{n+1}$, with $|\boldsymbol{\beta}|=\beta_{0}+\beta_{1}+\cdots+\beta_{n}$. Given that $\lambda_{j} \in \mathscr{P}_{1}$, we have that $\lambda^{\boldsymbol{\beta}}=\lambda_{0}^{\beta_{0}} \lambda_{1}^{\beta_{1}} \cdots \lambda_{n}^{\beta_{n}} \in \mathscr{P}_{|\boldsymbol{\beta}|}$, for every $\boldsymbol{\beta} \in \mathbb{N}^{n+1}$. Then, it is straightforward to show that

$$
\mathscr{P}_{d}=\operatorname{span}\left\{\lambda^{\beta}: \boldsymbol{\beta} \in \mathbb{N}^{n+1},|\boldsymbol{\beta}|=d\right\} .
$$

On the other hand, we can use basic rules (1.9) for representing the invariant part of each polynomial $\lambda^{\beta}$ as

$$
q_{\boldsymbol{\beta}}(\mathbf{x})=R\left(\lambda^{\beta} ; \mathbf{x}\right) .
$$


Then,

$$
\mathscr{P}_{d}^{*}=\operatorname{span}\left\{q_{\boldsymbol{\beta}}: \boldsymbol{\beta} \in \mathbb{N}^{n+1},|\boldsymbol{\beta}|=d\right\}
$$

and hence, denoting by

$$
A(n, d)=\operatorname{card}\left\{\boldsymbol{\beta} \in \mathbb{N}^{n+1}:|\boldsymbol{\beta}|=d, \beta_{0} \geq \beta_{1} \geq \cdots \geq \beta_{n}\right\},
$$

we have

$$
\operatorname{dim} \mathscr{P}_{d}^{*}=A(n, d), n, d \in \mathbb{N}, n \geq 1 .
$$

Now it is important to guarantee that invariance and homogeneity are compatible. This will be done through the following

Definition 6. A nondegenerate $n$-simplex $T_{n}$ is said to be centered at the origin when its barycenter coincides with the origin of coordinates, that is, when

$$
\lambda_{j}(0,0, \ldots, 0)=\frac{1}{n+1}, j=0, \ldots, n .
$$

Note that when the simplex is centered at the origin we have

$$
\mathbf{s}(0,0, \ldots, 0)=(0,0, \ldots, 0), \forall \mathbf{s} \in \mathscr{G}_{n},
$$

which ensures that (1.2) can be satisfied by a homogeneous polynomial $p$.

Lemma 1. For every $n, d \in \mathbb{N}, n \geq 1$, let $A(n, d)$ be given by (3.5) and let $\lfloor d / 2\rfloor$ stand for the integer part of $d / 2$. Then,

$$
\begin{aligned}
& A(1, d)=1+\lfloor d / 2\rfloor \text { for every } d \in \mathbb{N}, \\
& A(n, d)=A(n-1, d) \text { if } n \geq 2 \text { and } d \leq n, \\
& A(n, d)=A(n-1, d)+A(n, d-n-1) \\
& \text { if } n \geq 2 \text { and } d \geq n+1 .
\end{aligned}
$$

Proof. It is clear that

$$
A(1, d)=\operatorname{card}\left\{\left(\beta_{0}, \beta_{1}\right) \in \mathbb{N}^{2}: \beta_{0} \geq \beta_{1}, \beta_{0}+\beta_{1}=d\right\}=1+\lfloor d / 2\rfloor .
$$

Now, let $n \geq 2$ and $d \leq n$. This implies $\beta_{n}=0$ in (3.5) and therefore

$$
\begin{aligned}
A(n, d) & =\operatorname{card}\left\{\left(\beta_{0}, \ldots, \beta_{n-1}, 0\right) \in \mathbb{N}^{n+1}: \beta_{0} \geq \cdots \geq \beta_{n-1} \geq 0, \sum_{i=0}^{n-1} \beta_{i}=d\right\} \\
& =\operatorname{card}\left\{\left(\beta_{0}, \ldots, \beta_{n-1}\right) \in \mathbb{N}^{n}: \beta_{0} \geq \cdots \geq \beta_{n-1}, \sum_{i=0}^{n-1} \beta_{i}=d\right\} \\
& =A(n-1, d) .
\end{aligned}
$$

Finally, when $n \geq 2$ and $d \geq n+1$ we can consider two possibilities in (3.5). Either $\beta_{n}=0$ or $\beta_{n} \geq 1$. Then,

$$
\begin{aligned}
A(n, d)= & A(n-1, d) \\
& +\operatorname{card}\left\{\left(\beta_{0}, \ldots, \beta_{n}\right) \in \mathbb{N}^{n+1}: \beta_{0} \geq \cdots \geq \beta_{n} \geq 1, \sum_{i=0}^{n} \beta_{i}=d\right\}
\end{aligned}
$$


and calling $\gamma_{i}=\beta_{i}-1$, we have

$$
\begin{aligned}
A(n, d)= & A(n-1, d) \\
& +\operatorname{card}\left\{\left(\gamma_{0}, \ldots, \gamma_{n}\right) \in \mathbb{N}^{n+1}: \gamma_{0} \geq \cdots \geq \gamma_{n}, \sum_{i=0}^{n} \gamma_{i}=d-n-1\right\} \\
= & A(n-1, d)+A(n, d-n-1) .
\end{aligned}
$$

Lemma 2. Given a nondegenerate simplex $T_{n}$ in $\mathbb{R}^{n}$, let $\mathbf{u}=\left(u_{1}, \ldots, u_{n}\right)$ be the map u: $\mathbb{R}^{n} \rightarrow \mathbb{R}^{n}$ defined by

$$
u_{k}(\mathbf{x})=\sum_{j=0}^{n}\left[\lambda_{j}(\mathbf{x})-\lambda_{j}(0,0, \ldots, 0)\right]^{k+1}, k=1, \ldots, n,
$$

and, for every multi-index $\boldsymbol{\alpha}=\left(\alpha_{1}, \ldots, \alpha_{n}\right) \in \mathbb{N}^{n}$, let $\mathbf{u}^{\alpha}$ be the polynomial $\mathbf{u}^{\alpha}=u_{1}^{\alpha_{1}} \cdots u_{n}^{\alpha_{n}}$. Then

(a) The interior of the set $U=\mathbf{u}\left(\mathbb{R}^{n}\right)$ is not empty.

(b) The set of polynomials $\left\{\mathbf{u}^{\alpha}: \boldsymbol{\alpha} \in \mathbb{N}^{n}\right\}$ is linearly independent in $\mathscr{P}$.

Proof. First of all, denote

$$
\mu_{j}(\mathbf{x})=\lambda_{j}(\mathbf{x})-\lambda_{j}(0,0, \ldots, 0), j=0, \ldots, n .
$$

Then, given that $\sum_{j=0}^{n} \lambda_{j}(\mathbf{x})=1$, we have $\sum_{j=0}^{n} \mu_{j}(\mathbf{x})=0$ and hence $\mu_{0}(\mathbf{x})=$ $\sum_{j=1}^{n}-\mu_{j}(\mathbf{x})$. Therefore, we can write

$$
\mathbf{u}(\mathbf{x})=\mathbf{z}(\boldsymbol{\mu}(\mathbf{x})),
$$

where $\boldsymbol{\mu}=\left(\mu_{1}, \ldots, \mu_{n}\right), \mathbf{z}=\left(z_{1}, \ldots, z_{n}\right)$ and

$$
z_{k}(\boldsymbol{\mu})=\sum_{j=1}^{n} \mu_{j}^{k+1}+\left(\sum_{j=1}^{n}-\mu_{j}\right)^{k+1} \quad, k=1, \ldots, n .
$$

Now the Jacobian of the map $\mathbf{u}$, say $\left|\frac{\partial \mathbf{u}}{\partial \mathbf{x}}\right|$, can be written as

$$
\left|\frac{\partial \mathbf{u}}{\partial \mathbf{x}}\right|=\left|\frac{\partial \mathbf{z}}{\partial \boldsymbol{\mu}}\right|\left|\frac{\partial \boldsymbol{\mu}}{\partial \mathbf{x}}\right| \text {. }
$$

Note that $\frac{\partial \mu_{j}}{\partial x_{i}}=\frac{\partial \lambda_{j}}{\partial x_{i}}$ and, given that the simplex $T_{n}$ is nondegenerate, this implies that the Jacobian $\left|\frac{\partial \mu}{\partial \mathbf{x}}\right|$ is constant and different from zero. On the other hand, differentiating (3.13), we get

$$
\frac{\partial z_{k}}{\partial \mu_{i}}=(k+1)\left(\mu_{i}^{k}-\mu_{0}^{k}\right), i, k=1, \ldots, n
$$

But

$$
\left|\begin{array}{ccc}
\mu_{1}-\mu_{0} & \ldots & \mu_{n}-\mu_{0} \\
\mu_{1}^{2}-\mu_{0}^{2} & \ldots & \mu_{n}^{2}-\mu_{0}^{2} \\
\vdots & \ddots & \vdots \\
\mu_{1}^{n}-\mu_{0}^{n} & \ldots & \mu_{n}^{n}-\mu_{0}^{n}
\end{array}\right|=\left|\begin{array}{cccc}
1 & 1 & \ldots & 1 \\
\mu_{0} & \mu_{1} & \ldots & \mu_{n} \\
\mu_{0}^{2} & \mu_{1}^{2} & \ldots & \mu_{n}^{2} \\
\vdots & \vdots & \ddots & \vdots \\
\mu_{0}^{n} & \mu_{1}^{n} & \ldots & \mu_{n}^{n}
\end{array}\right|
$$


and therefore

$$
\left|\frac{\partial \mathbf{z}}{\partial \boldsymbol{\mu}}\right|=(n+1) ! \prod_{j>i}\left|\mu_{j}-\mu_{i}\right| .
$$

In conclusion, we have proved that the Jacobian $\left|\frac{\partial \mathbf{u}}{\partial \mathbf{x}}\right|$ is different from zero in all points of $\mathbb{R}^{n}$ except in those belonging to the hyperplanes defined by

$$
\mu_{j}(\mathbf{x})-\mu_{i}(\mathbf{x})=0, i, j=0, \ldots, n, j>i .
$$

Now, use the Inverse Function Theorem and the result (a) follows immediately.

To prove (b), suppose that for a finite set $I \subset \mathbb{N}^{n}$ we have a polynomial $p(\mathbf{x})=\sum_{\boldsymbol{\alpha} \in I} a_{\boldsymbol{\alpha}} \mathbf{u}(\mathbf{x})^{\alpha}$ such that $p(\mathbf{x})=0$ for every $\mathbf{x} \in \mathbb{R}^{n}$. This means that the polynomial $q(\mathbf{u})=\sum_{\alpha \in I} a_{\alpha} \mathbf{u}^{\alpha}$ vanishes for all $\mathbf{u} \in U$. Given that the interior of $U$ is not empty, and that the monomials $\mathbf{u}^{\alpha}$ are linearly independent when considered as polynomials in the variables $u_{1}, \ldots, u_{n}$, this implies $a_{\alpha}=0$ for every $\alpha \in I$, and the result follows.

Lemma 3. For every $n, d \in \mathbb{N}, n \geq 1$, let $A(n, d)$ be given by (3.5) and let

$$
B(n, d)=\operatorname{card}\left\{\boldsymbol{\alpha} \in \mathbb{N}^{n}: \sum_{i=1}^{n}(i+1) \alpha_{i} \leq d\right\} .
$$

Then, $B(n, d)=A(n, d)$.

Proof. Given that the relations (3.8), (3.9) and (3.10) determine the value of $A(n, d)$ for all $n, d \in \mathbb{N}, n \geq 1$, it is sufficient to show that also $B(n, d)$ satisfies those relations. For $n=1$, we have $B(1, d)=\operatorname{card}\left\{\alpha_{1}: 2 \alpha_{1} \leq d\right\}=$ $1+\lfloor d / 2\rfloor$. On the other hand, when $n \geq 2$ the values that $\alpha_{n}$ can take in (3.14) are $\alpha_{n}=0,1, \ldots,\lfloor d /(n+1)\rfloor$. Then, fixing $\alpha_{n}=k$, we have

$$
\begin{aligned}
\operatorname{card}\left\{\left(\alpha_{1}, \ldots, \alpha_{n-1}, k\right)\right. & \left.\in \mathbb{N}^{n}: \sum_{i=1}^{n-1}(i+1) \alpha_{i}+(n+1) k \leq d\right\} \\
= & \operatorname{card}\left\{\alpha \in \mathbb{N}^{n-1}: \sum_{i=1}^{n-1}(i+1) \alpha_{i} \leq d-k(n+1)\right\},
\end{aligned}
$$

and hence

$$
B(n, d)=\sum_{k=0}^{\lfloor d /(n+1)\rfloor} B(n-1, d-k(n+1)),
$$

which proves that $B(n, d)=B(n-1, d)$ when $d \leq n$. Finally, when $d \geq n+1$, we can use $(3.15)$ to write

$$
\begin{aligned}
B(n, d-(n+1))=\sum_{k=0}^{\lfloor d /(n+1)\rfloor-1} B(n-1, & d-(k+1)(n+1)) \\
& =\sum_{k=1}^{\lfloor d /(n+1)\rfloor} B(n-1, d-k(n+1)) .
\end{aligned}
$$

Then we have that

$$
B(n, d)-B(n, d-(n+1))=B(n-1, d),
$$

which completes the proof. 
Theorem 2. Let a nondegenerate simplex $T_{n}$ be centered at the origin and, for every $\boldsymbol{\alpha} \in \mathbb{N}^{n}$, let $\mathbf{u}^{\alpha}=u_{1}^{\alpha_{1}} u_{2}^{\alpha_{2}} \cdots u_{n}^{\alpha_{n}}$, where $u_{k}=u_{k}(\mathbf{x}), k=1, \ldots, n$, are the polynomials defined by (3.11). Then

$$
\begin{aligned}
& \mathscr{H}_{d}^{*}=\operatorname{span}\left\{\mathbf{u}^{\alpha}: \alpha \in \mathbb{N}^{n}, \sum_{i=1}^{n}(i+1) \alpha_{i}=d\right\}, \\
& \mathscr{P}_{d}^{*}=\operatorname{span}\left\{\mathbf{u}^{\alpha}: \alpha \in \mathbb{N}^{n}, \sum_{i=1}^{n}(i+1) \alpha_{i} \leq d\right\}, \\
& \mathscr{P}^{*}=\operatorname{span}\left\{\mathbf{u}^{\alpha}: \alpha \in \mathbb{N}^{n}\right\} .
\end{aligned}
$$

Proof. It follows from (3.11) that each $u_{k}$ is a homogeneous polynomial of degree $d=(k+1)$. Moreover, given that the simplex $T_{n}$ is centered at the origin, we can write $(3.11)$ as

$$
u_{k}(\mathbf{x})=\sum_{j=0}^{n}\left[\lambda_{j}(\mathbf{x})-\frac{1}{n+1}\right]^{k+1}, k=1, \ldots, n,
$$

which shows that the polynomials $u_{k}(\mathbf{x})$ are also invariant under permutations of the barycentric coordinates $\lambda_{j}(\mathbf{x})$. Therefore, we have that each $\mathbf{u}^{\alpha}$ is a homogeneous and invariant polynomial of degree $d=\sum_{i=1}^{n}(i+1) \alpha_{i}$. On the other hand, using Lemma 3, we have that

$$
\operatorname{card}\left\{\mathbf{u}^{\alpha}: \alpha \in \mathbb{N}^{n}, \sum_{i=1}^{n}(i+1) \alpha_{i} \leq d\right\}=\operatorname{dim} \mathscr{P}_{d}^{*},
$$

since the polynomials $\mathbf{u}^{\alpha}, \alpha \in \mathbb{N}^{n}$, are linearly independent (Lemma 2). Now (3.17), and hence (3.16) and (3.18), follow immediately.

\section{CONSISTENCY CONDITIONS}

We now return to the problem of constructing an invariant quadrature rule (2.9) with a given degree of precision $d$. In what follows the simplex $T_{n}$ will be supposed to be centered at the origin, and we shall use the basis of $\mathscr{P}_{d}^{*}$ given by (3.17). Therefore, we shall refer to

$$
Q\left(\mathbf{u}^{\alpha}\right)-I\left(\mathbf{u}^{\alpha}\right)=0, \quad \alpha \in \mathbb{N}^{n}: \sum_{i=1}^{n}(i+1) \alpha_{i} \leq d
$$

as the moment equations that the rule must fit to have degree of precision $d$. Note that the rule structure $\left(K_{0}, K_{1}, \ldots, K_{M_{n}}\right)$ determines the functional dependence on the unknowns (free parameters) in the nonlinear system of moment equations.

On the other hand, the number of nodes of the rule and the number of unknowns of the system of moment equations are given by (2.10) and (2.11), respectively, where the values of $\nu_{i}$ and $\left(r_{i}+1\right)$ for each type of basic rule are listed in Tables 1 and 2. This situation suggests that there are types of basic rules that are better than others, in the sense that they produce more free parameters with a smaller number of nodes. Nevertheless, it is not possible to 
construct an invariant quadrature rule with a given degree of precision if we choose arbitrarily the numbers $K_{i}$ which define the structure. This is because the existence of a solution of (4.1) requires a certain minimum number of basic rules of each type. These restrictions on the numbers $K_{i}$ are called consistency conditions and were introduced in [14, 18 and 19] for fully symmetric multidimensional quadrature rules. In this section we derive general consistency conditions for invariant quadrature rules for the $n$-dimensional simplex $T_{n}$, in a convenient and systematic form.

Before deriving the consistency conditions, let us try to clarify the meaning of this concept. We consider the system of moment equations (4.1) and note that Lemma 2 guarantees that there is no linear dependence between these equations. Under the hypothesis that there is no other form of algebraic dependence between the moment equations, it is commonly assumed that a necessary condition for this nonlinear system to have a solution is that the number of unknowns of the system be greater or equal than the number of equations, i.e.,

$$
\sum_{i=0}^{M_{n}}\left(r_{i}+1\right) K_{i} \geq \operatorname{dim} \mathscr{P}_{d}^{*} .
$$

However, we can also obtain linear combinations of equations (4.1) that make some of the unknowns disappear. These kinds of subsystems will be obtained by applying the equation $Q(p)-I(p)=0$ to polynomials $p$ belonging to some special subspaces of $\mathscr{P}_{d}^{*}$. When the equations of these subsystems are linearly independent, a condition that is guaranteed by the linear independence of the polynomials $p$ used to obtain them, it is natural to impose, as a necessary condition for the existence of solutions, that the number of unknowns that remain in the subsystem be greater or equal than the number of equations of the subsystem. In order to translate these ideas into conditions on the rule structure of a quadrature rule, that is, into inequalities similar to (4.2), we consider, for a given $n$, the classes $\mathscr{C}_{i}, i=0, \ldots, M_{n}$, with the order relation $\prec$ defined in $\S 1$.

Definition 7. A set $J \subset\left\{0, \ldots, M_{n}\right\}$ is said to be a consistency set if it has the property that for every $i, j \in J$ such that $i \neq j, \mathscr{C}_{i} \nprec \mathscr{C}_{j}$ and $\mathscr{C}_{j} \nprec \mathscr{C}_{i}$. That is, a consistency set is a set of indices of classes that are not related by $\prec$.

Definition 8. Given a consistency set $J$, the null space $\mathscr{P}_{d}^{*}(J)$ is the linear space of invariant polynomials given by

$$
\mathscr{P}_{d}^{*}(J)=\left\{p \in \mathscr{P}_{d}^{*}: \forall j \in J, \forall \mathbf{x} \in \mathscr{C}_{j}, p(\mathbf{x})=0\right\} .
$$

Now, for every consistency set $J$ denote by $J^{\prime}$ the set $J^{\prime} \subset\left\{0,1, \ldots, M_{n}\right\}$ defined by

$$
J^{\prime}=\left\{i \notin J: \forall j \in J, \mathscr{C}_{i} \nprec \mathscr{C}_{j}\right\}
$$

and consider any equation of the form

$$
Q(p)-I(p)=0, p \in \mathscr{P}_{d}^{*}(J),
$$

that is obtained as a linear combination of the moment equations (4.1). Note that in such an equation the parameters that correspond to basic rules of types $\mathscr{C}_{i}$ such that $\mathscr{C}_{i}=\mathscr{C}_{j}$ or $\mathscr{C}_{i} \prec \mathscr{C}_{j}$ for some $j \in J$ do not appear. Given that 
the maximum number of linearly independent equations of the form (4.5) is equal to the dimension of $\mathscr{P}_{d}^{*}(J)$, we have a consistency condition associated with every consistency set $J$ and hence the following

Definition 9. A rule structure $\left(K_{0}, K_{1}, \ldots, K_{M_{n}}\right)$ is $d$-consistent when the consistency condition

$$
\sum_{i \in J^{\prime}}\left(r_{i}+1\right) K_{i} \geq \operatorname{dim} \mathscr{P}_{d}^{*}(J)
$$

is satisfied for every consistency set $J \subset\left\{0,1, \ldots, M_{n}\right\}$.

We note that, taking $J=\varnothing$, we have $\mathscr{P}_{d}^{*}(J)=\mathscr{P}_{d}^{*}$ and $J^{\prime}=\{0,1, \ldots$, $\left.M_{n}\right\}$. Hence, the first consistency condition we obtain is (4.2). On the other hand, given that $\mathscr{C}_{M_{n}}=[1,1, \ldots, 1]=\mathbb{R}^{n}$, taking $J=\left\{M_{n}\right\}$, we have $J^{\prime}=\varnothing$ and $\operatorname{dim} \mathscr{P}_{d}^{*}(J)=0$. Hence, the corresponding consistency condition reduces to the trivial inequality $0 \geq 0$.

Now, we need to use several spaces of polynomials whose set of variables is different from $\mathbf{x}=\left(x_{1}, \ldots, x_{n}\right)$. As usual, we shall refer to these spaces by specifying the set of variables between brackets, that is, denoting them as $\mathscr{P}\left[y_{1}, \ldots, y_{r}\right], \mathscr{P}_{d}\left[y_{1}, \ldots, y_{r}\right]$ or $\mathscr{H}_{d}\left[y_{1}, \ldots, y_{r}\right]$, for instance.

For every $i=0, \ldots, M_{n}$, let $\mathscr{C}_{i}=\left[m_{0}, m_{1}, \ldots, m_{r_{i}}\right]$, and let $V_{i}$ be the $r_{i}$-dimensional affine manifold such that $\left\{\mathbf{s}\left(V_{i}\right): \mathbf{s} \in \mathscr{G}_{n}\right\}$ coincides with the set of points of class $\mathscr{C}_{i}$. According to Definition 3 , each point $\mathbf{x} \in V_{i}$ has a barycentric representation

$$
\lambda(\mathbf{x})=(\overbrace{a_{0}, \ldots, a_{0}}^{m_{0} \text { times }}, \overbrace{a_{1}, \ldots, a_{1}}^{m_{1} \text { times }}, \ldots, \overbrace{a_{r_{i}}, \ldots, a_{r_{i}}}^{m_{r_{i}} \text { times }}),
$$

with $\sum_{j=0}^{r_{i}} m_{j} a_{j}=1$. If we call

$$
y_{j}=a_{j}-\frac{1}{n+1}, j=0, \ldots, r_{i},
$$

with $\sum_{j=0}^{r_{i}} m_{j} y_{j}=0$, we can translate $(4.7)$ into

$$
\mathbf{x}=\mathbf{h}_{i}\left(y_{1}, \ldots, y_{r_{i}}\right),
$$

and $V_{i}$ can be written as

$$
V_{i}=\left\{\mathbf{x}=\mathbf{h}_{i}\left(y_{1}, \ldots, y_{r_{i}}\right):\left(y_{1}, \ldots, y_{r_{i}}\right) \in \mathbb{R}^{r_{i}}\right\}
$$

Then, for every $i=1, \ldots, M_{n}$, we can define a linear map, usually called comorphism, $w_{i}: \mathscr{P}^{*} \rightarrow \mathscr{P}\left[y_{1}, \ldots, y_{r_{t}}\right]$ given by

$$
w_{i}(p)\left(y_{1}, \ldots, y_{r_{1}}\right)=p\left(\mathbf{h}_{i}\left(y_{1}, \ldots, y_{r_{1}}\right)\right), p \in \mathscr{P}^{*} .
$$

For $i=0$, we have $\mathscr{C}_{0}=[n+1]$ and $r_{0}=0$. Then, $w_{0}: \mathscr{P}^{*} \rightarrow \mathbb{R}$ is given by $w_{0}(p)=p(0, \ldots, 0), p \in \mathscr{P}^{*}$.

On the other hand, given that $\mathscr{P}^{*}=\operatorname{span}\left\{\mathbf{u}^{\alpha}: \alpha \in \mathbb{N}^{n}\right\}$ (see Theorem 2), we can define the comorphisms $w_{i}, i=0, \ldots, M_{n}$, by calculating $w_{i}\left(\mathbf{u}^{\alpha}\right)$ for $\alpha \in \mathbb{N}^{n}$. It is clear that

$$
w_{0}\left(\mathbf{u}^{\boldsymbol{\alpha}}\right)= \begin{cases}1 & \text { if } \boldsymbol{\alpha}=(0, \ldots, 0) \\ 0 & \text { if } \boldsymbol{\alpha} \neq(0, \ldots, 0)\end{cases}
$$


and, for $i=1, \ldots, M_{n}$ and $k=1, \ldots, n$, using (3.19), we have

$$
w_{i}\left(u_{k}\right)\left(y_{1}, \ldots, y_{r_{i}}\right)=\sum_{j=0}^{r_{i}} m_{j} y_{j}^{k+1}
$$

where $y_{0}=-\frac{1}{m_{0}} \sum_{j=1}^{r_{i}} m_{j} y_{j}$. Therefore,

$$
w_{i}\left(u_{k}\right)\left(y_{1}, \ldots, y_{r_{i}}\right)=\frac{(-1)^{k+1}}{m_{0}^{k}}\left(\sum_{j=1}^{r_{i}} m_{j} y_{j}\right)^{k+1}+\sum_{j=1}^{r_{i}} m_{j} y_{j}^{k+1}
$$

and

$$
w_{i}\left(\mathbf{u}^{\boldsymbol{\alpha}}\right)=\prod_{k=1}^{n}\left(w_{i}\left(u_{k}\right)\right)^{\alpha_{k}}, \quad \boldsymbol{\alpha} \in \mathbb{N}^{n}, i=1, \ldots, M_{n} .
$$

Note that in view of $(3.16),(4.14)$ and (4.15), it is straightforward to prove that

$$
p \in \mathscr{H}_{d}^{*} \Longrightarrow w_{i}(p) \in \mathscr{H}_{d}\left[y_{1}, \ldots, y_{r_{i}}\right], i=1, \ldots, M_{n} .
$$

Now, for every consistency set $J=\left\{i_{1}, \ldots, i_{s}\right\}, J \neq \varnothing$, let $\mathbf{w}_{J}$ be the map

$$
\mathbf{w}_{J}: \mathscr{P}^{*} \rightarrow \prod_{i \in J} \mathscr{P}\left[y_{1}, \ldots, y_{r_{i}}\right]
$$

defined by

$$
\mathbf{w}_{J}(p)=\left(w_{i_{1}}(p), \ldots, w_{i_{s}}(p)\right), p \in \mathscr{P}^{*} .
$$

Then, it is clear that the corresponding null space $\mathscr{P}_{d}^{*}(J)$ can be written as

$$
\mathscr{P}_{d}^{*}(J)=\left\{p \in \mathscr{P}_{d}^{*}: \mathbf{w}_{J}(p)=(0, \ldots, 0)\right\} .
$$

Lemma 4. For every consistency set $J \neq \varnothing$,

$$
\operatorname{dim} \mathbf{w}_{J}\left(\mathscr{P}_{d}^{*}\right)=\sum_{k=0}^{d} \operatorname{dim} \mathbf{w}_{J}\left(\mathscr{H}_{k}^{*}\right) .
$$

Proof. When $J=\{0\}$, we have $\mathbf{w}_{J}=w_{0}$. Then, using (4.12), we easily see that $\operatorname{dim} w_{0}\left(\mathscr{P}_{d}^{*}\right)=1, \operatorname{dim} w_{0}\left(\mathscr{H}_{0}^{*}\right)=1$ and $\operatorname{dim} w_{0}\left(\mathscr{H}_{k}^{*}\right)=0$ for $k \geq 1$. When $J \neq\{0\}$, we have that $0 \notin J$ (see Definition 7). In this case, we prove that

$$
\mathbf{w}_{J}\left(\mathscr{P}_{d}^{*}\right)=\mathbf{w}_{J}\left(\mathscr{H}_{0}^{*}\right) \oplus \cdots \oplus \mathbf{w}_{J}\left(\mathscr{H}_{d}^{*}\right)
$$

Given that $\mathbf{w}_{J}$ is linear and $\mathscr{P}_{d}^{*}=\mathscr{H}_{0}^{*} \oplus \cdots \oplus \mathscr{H}_{d}^{*}$, it is clear that for every $p \in \mathscr{P}_{d}^{*}$, we have $\mathbf{w}_{J}(p)=\sum_{k=0}^{d} \mathbf{w}_{J}\left(p_{k}\right)$, where $\mathbf{w}_{J}\left(p_{k}\right) \in \mathbf{w}_{J}\left(\mathscr{H}_{k}^{*}\right)$ for $k=0, \ldots, d$. On the other hand, let $p_{k} \in \mathscr{H}_{k}^{*}, k=0, \ldots, d$, such that $\sum_{k=0}^{d} \mathbf{w}_{J}\left(p_{k}\right)=0$. Then, $\sum_{k=0}^{d} w_{i}\left(p_{k}\right)=0$ for every $i \in J$. But (4.16) guarantees that $w_{i}\left(p_{k}\right) \in \mathscr{H}_{k}\left[y_{1}, \ldots, y_{r_{i}}\right]$ for $k=0, \ldots, d$, and this implies that $w_{i}\left(p_{0}\right), \ldots, w_{i}\left(p_{d}\right)$ are linearly independent. Therefore, we must have $w_{i}\left(p_{k}\right)=0, k=0, \ldots, d, i \in J$. That is, $\mathbf{w}_{J}\left(p_{k}\right)=0$ for $k=$ $0, \ldots, d$. 
Theorem 3. Let the simplex $T_{n}$ be centered at the origin and let $J \neq \varnothing$ be a consistency set. Then, for every $d \in \mathbb{N}$,

$$
\operatorname{dim} \mathscr{P}_{d}^{*}(J)=A(n, d)-\sum_{k=0}^{d} \operatorname{dim} \mathbf{w}_{J}\left(\mathscr{H}_{k}^{*}\right) .
$$

Proof. Let $\mathbf{w}_{J} \mid \mathscr{P}_{d}^{*}$ denote the restriction of $\mathbf{w}_{J}$ to $\mathscr{P}_{d}^{*}$. From (4.19) it is obvious that the null space $\mathscr{P}_{d}^{*}(J)$ is the kernel of $\mathbf{w}_{J}{\mid \mathscr{P}_{d}^{*}}^{\text {. But }} \mathbf{w}_{J}{\mid \mathscr{P}_{d}^{*}}_{d}$ is a linear map, and hence

$$
\operatorname{dim} \mathscr{P}_{d}^{*}=\operatorname{dim} \operatorname{Ker}\left(\mathbf{w}_{J}{\mid \mathscr{P}_{d}^{*}}\right)+\operatorname{dim} \operatorname{Im}\left(\mathbf{w}_{J}{\mid \mathscr{P}_{d}^{*}}\right),
$$

that is,

$$
A(n, d)=\operatorname{dim} \mathscr{P}_{d}^{*}(J)+\operatorname{dim} \mathbf{w}_{J}\left(\mathscr{P}_{d}^{*}\right) .
$$

Now, use Lemma 4 , and the result follows.

\section{ON THE COMPUTATION OF CONSISTENT STRUCTURES}

In this section we describe the more relevant aspects of the method we have used for computing optimal $d$-consistent structures, that is, $d$-consistent structures with a minimal number of nodes. We can also use a similar procedure for computing $d$-consistent structures that have a number of nodes that is near the minimal one. We shall refer to this kind of structures as quasi-optimal.

First of all, for a given range of values of $n$ and $d$, we use the recurrence relations given in Lemma 1 for calculating the numbers $A(n, d)$ or $\operatorname{dim} \mathscr{P}_{d}^{*}$ and store them in a file. For every dimension $n$, the set of classes $\left\{\mathscr{C}_{i}, i \stackrel{d=}{=}\right.$ $\left.0, \ldots, M_{n}\right\}$ is obtained, starting with the class $\mathscr{C}_{M_{n}}=[1,1, \ldots, 1]$, by using the order relation $\prec$, with the concept of contracted version explained in the comments to Definition 3. At the same time we construct the relation tree, with branches connecting the classes related by $\prec$, and store it as a matrix $\Psi=\left(\psi_{i j}\right)$ given by

$$
\psi_{i j}= \begin{cases}1 & \text { if } \mathscr{C}_{i} \prec \mathscr{C}_{j}, \\ 0 & \text { if } \mathscr{C}_{i} \nprec \mathscr{C}_{j} .\end{cases}
$$

For each class we also calculate the numbers $\nu_{i}$ and $r_{i}$. Using the matrix $\Psi$, we easily obtain all the consistency sets $J$ and the corresponding $J^{\prime}$, which allows us to construct the left-hand sides of the consistency conditions (4.6).

Now, for a given $n$, the next step is to obtain the right-hand sides of (4.6). We first note that if $J=\varnothing$, then $\operatorname{dim} \mathscr{P}_{d}^{*}(J)=A(n, d)$. For other consistency sets $J \neq \varnothing$, Theorem 3 shows that it is sufficient to calculate $\operatorname{dim} \mathbf{w}_{J}\left(\mathscr{H}_{d}^{*}\right)$ for the desired range of values of $d$. It is well known that $\operatorname{dim} \mathbf{w}_{J}\left(\mathscr{H}_{d}^{*}\right)$ coincides with the rank of the matrix that represents the linear map $\left.\mathbf{w}_{J}\right|_{\mathscr{H}_{d}^{*}}$, i.e., the restriction of $\mathbf{w}_{J}$ to $\mathscr{H}_{d}^{*}$. When the consistency set has only one element, say $J=\{i\}$, this matrix consists of the coefficients of the polynomials $w_{i}\left(\mathbf{u}^{\boldsymbol{\alpha}}\right)$, for the $\alpha \in \mathbb{N}^{n}$ such that $\sum_{i=1}^{n}(i+1) \alpha_{i}=d$, when they are written as linear combinations of the standard basis of monomials of the space $\mathscr{H}_{d}\left[y_{1}, \ldots, y_{r_{i}}\right]$. When $J=\left\{i_{1}, \ldots, i_{s}\right\}$, then $\mathbf{w}_{J}=\left(w_{i_{1}}, \ldots, w_{i_{s}}\right)$ and the corresponding matrix associated with $J$ is the matrix $\left(E_{i_{1}}\left|E_{i_{2}}\right| \cdots \mid E_{i_{s}}\right)$, where each $E_{i_{j}}$ stands for the matrix associated with $J=\left\{i_{j}\right\}$ as shown before. We illustrate this with two examples for $n=3$ and $d=6$. 
Example 1. Consider the consistency set $J=\{3\}$ corresponding to the class $\mathscr{C}_{3}=[2,1,1]$. According to $(4.14)$ the polynomials $w_{3}\left(u_{k}\right)$ are

$$
\begin{aligned}
& w_{3}\left(u_{1}\right)=y_{1}^{2}+y_{2}^{2}+\frac{1}{2}\left(y_{1}+y_{2}\right)^{2}=\frac{1}{2}\left(3 y_{1}^{2}+2 y_{1} y_{2}+3 y_{2}^{2}\right), \\
& w_{3}\left(u_{2}\right)=y_{1}^{3}+y_{2}^{3}-\frac{1}{4}\left(y_{1}+y_{2}\right)^{3}=\frac{1}{4}\left(3 y_{1}^{3}-3 y_{1}^{2} y_{2}-3 y_{1} y_{2}^{2}+3 y_{2}^{3}\right), \\
& w_{3}\left(u_{3}\right)=y_{1}^{4}+y_{2}^{4}+\frac{1}{8}\left(y_{1}+y_{2}\right)^{4}=\frac{1}{8}\left(9 y_{1}^{4}+4 y_{1}^{3} y_{2}+6 y_{1}^{2} y_{2}^{2}+4 y_{1} y_{2}^{3}+9 y_{2}^{4}\right) .
\end{aligned}
$$

Then, the images of the corresponding homogeneous polynomials $\mathbf{u}^{\alpha}$ of degree $d=6$ are

$$
\begin{aligned}
w_{3}\left(u_{1}^{3}\right) & =\frac{1}{8}\left(27 y_{1}^{6}+54 y_{1}^{5} y_{2}+117 y_{1}^{4} y_{2}^{2}+116 y_{1}^{3} y_{2}^{3}+117 y_{1}^{2} y_{2}^{4}+54 y_{1} y_{2}^{5}+27 y_{2}^{6}\right), \\
w_{3}\left(u_{2}^{2}\right) & =\frac{1}{16}\left(9 y_{1}^{6}-18 y_{1}^{5} y_{2}-9 y_{1}^{4} y_{2}^{2}+36 y_{1}^{3} y_{2}^{3}-9 y_{1}^{2} y_{2}^{4}-18 y_{1} y_{2}^{5}+9 y_{2}^{6}\right), \\
w_{3}\left(u_{1} u_{3}\right) & =\frac{1}{16}\left(27 y_{1}^{6}+30 y_{1}^{5} y_{2}+53 y_{1}^{4} y_{2}^{2}+36 y_{1}^{3} y_{2}^{3}+53 y_{1}^{2} y_{2}^{4}+30 y_{1} y_{2}^{5}+27 y_{2}^{6}\right) .
\end{aligned}
$$

Therefore, the associated coefficient matrix is

$$
E=\frac{1}{16}\left(\begin{array}{ccccccc}
54 & 108 & 234 & 232 & 234 & 108 & 54 \\
9 & -18 & -9 & 36 & -9 & -18 & 9 \\
27 & 30 & 53 & 36 & 53 & 30 & 27
\end{array}\right)
$$

Given that $\operatorname{rank}(E)=3$, we have $\operatorname{dim} \mathbf{w}_{J}\left(\mathscr{H}_{6}^{*}\right)=3$.

Example 2. Consider now $J=\{1,2\}$, with $\mathscr{C}_{1}=[3,1]$ and $\mathscr{C}_{2}=[2,2]$. Then,

$$
\begin{array}{ll}
w_{1}\left(u_{1}\right)=y_{1}^{2}+\frac{1}{3} y_{1}^{2}=\frac{4}{3} y_{1}^{2}, & w_{2}\left(u_{1}\right)=2 y_{1}^{2}+2 y_{1}^{2}=4 y_{1}^{2}, \\
w_{1}\left(u_{2}\right)=y_{1}^{3}-\frac{1}{9} y_{1}^{3}=\frac{8}{9} y_{1}^{3}, & w_{2}\left(u_{2}\right)=2 y_{1}^{3}-2 y_{1}^{3}=0, \\
w_{1}\left(u_{3}\right)=y_{1}^{4}+\frac{1}{27} y_{1}^{4}=\frac{28}{27} y_{1}^{4}, & w_{2}\left(u_{3}\right)=2 y_{1}^{4}+2 y_{1}^{4}=4 y_{1}^{4} .
\end{array}
$$

Now, the images of the homogeneous polynomials $\mathbf{u}^{\alpha}$ of degree $d=6$ and their corresponding matrices are

$$
\begin{aligned}
w_{1}\left(u_{1}^{3}\right) & =\frac{64}{27} y_{1}^{6}, \\
w_{1}\left(u_{2}^{2}\right) & =\frac{64}{81} y_{1}^{6}, \\
w_{1}\left(u_{1} u_{3}\right) & =\frac{112}{81} y_{1}^{6},
\end{aligned} \quad E_{1}=\left(\begin{array}{rl}
\frac{64}{27} \\
\frac{64}{81} \\
\frac{112}{81}
\end{array}\right), \quad \begin{aligned}
w_{2}\left(u_{1}^{3}\right) & =64 y_{1}^{6}, \\
w_{2}\left(u_{2}^{2}\right) & =0, \\
w_{2}\left(u_{1} u_{3}\right) & =16 y_{1}^{6},
\end{aligned} \quad E_{2}=\left(\begin{array}{c}
64 \\
0 \\
16
\end{array}\right) .
$$

Then, the matrix associated with the consistency set $J$ is

$$
\left(E_{1} \mid E_{2}\right)=\left(\begin{array}{cc}
\frac{64}{27} & 64 \\
\frac{64}{81} . & 0 \\
\frac{112}{81} & 16
\end{array}\right), \quad \operatorname{rank}\left(E_{1} \mid E_{2}\right)=2,
$$

and hence, $\operatorname{dim} \mathbf{w}_{J}\left(\mathscr{H}_{6}^{*}\right)=2$.

In the general procedure the rank of these matrices is calculated by a standard singular value decomposition routine. Some savings can be effected in practice by taking into account some special situations. For example, when for a given degree $d$ we find a consistency set $J$ such that the rank of its associated matrix 
TABLE 3. Minimal number of nodes $\nu_{L}(n, d)$

\begin{tabular}{|l|r|r|r|r|r|r|r|}
\hline & $n=2$ & $n=3$ & $n=4$ & $n=5$ & $n=6$ & $n=7$ & $n=8$ \\
\hline$d=1$ & 1 & 1 & 1 & 1 & 1 & 1 & 1 \\
$d=2$ & 3 & 4 & 5 & 6 & 7 & 8 & 9 \\
$d=3$ & 4 & 5 & 6 & 7 & 8 & 9 & 10 \\
$d=4$ & 6 & 11 & 16 & 22 & 29 & 37 & 46 \\
$d=5$ & 7 & 14 & 21 & 28 & 36 & 45 & 55 \\
$d=6$ & 12 & 24 & 41 & 68 & 98 & 136 & 183 \\
$d=7$ & 13 & 30 & 55 & 84 & 140 & 192 & 255 \\
$d=8$ & 16 & 43 & 90 & 164 & 267 & 431 & 622 \\
$d=9$ & 19 & 52 & 120 & 210 & 351 & 578 & 835 \\
$d=10$ & 24 & 68 & 171 & 325 & 644 & 1076 & 1699 \\
$d=11$ & 27 & 81 & 206 & 470 & 848 & 1511 & \\
$d=12$ & 33 & 117 & 306 & 736 & 1456 & 2715 & \\
$d=13$ & 36 & 133 & 381 & 917 & 1911 & 3633 & \\
$d=14$ & 42 & 163 & 485 & 1272 & 2870 & & \\
$d=15$ & 46 & 190 & 616 & 1662 & 3816 & & \\
$d=16$ & 52 & 233 & 766 & 2218 & & & \\
$d=17$ & 58 & 266 & 931 & 2734 & & & \\
$d=18$ & 66 & 318 & 1161 & 3649 & & & \\
$d=19$ & 70 & 355 & 1396 & & & & \\
$d=20$ & 78 & 415 & 1750 & & & & \\
$d=21$ & 85 & 472 & 2060 & & & & \\
$d=22$ & 93 & 539 & 2421 & & & & \\
$d=23$ & 100 & 602 & 2826 & & & & \\
\hline
\end{tabular}

coincides with its number of rows, then we know that this rank holds for any other consistency set that contains $J$. On the other hand, some precautions must be taken when using a singular value decomposition routine for calculating the rank of matrices that can be very large. We have used 32 digits of accuracy and a standard test which takes into account the dimensions of the matrix, the biggest modulus of the singular values and the precision of the machine to decide whether a singular value is equal to, or different from, zero. In addition, the result is only accepted if there is a significant ratio (of at least $10^{10}$ ) between the smallest modulus of the nonzero singular values and the biggest modulus of the singular values considered zero. These precautions limit considerably the range of values of $n$ and $d$ that can be considered, but they are necessary to insure confidence that the computed ranks are correct. This is why some entries in Table 3 are missing.

Now, for obtaining an optimal $d$-consistent structure we have to solve a problem of integer linear programming in which the objective function to be minimized is the number of nodes given by (2.10), subject to the constraints 
TABLE 4. Optimal consistent structures for $T_{2}$ and $T_{3}$

\begin{tabular}{|r|r|rrr|r|r|}
\hline$n$ & $d$ & $K_{0}$ & $K_{1}$ & $K_{2}$ & $N_{P}(Q)$ & $\nu(Q)$ \\
\hline 2 & 1 & 1 & & 1 & 1 \\
2 & 2 & 0 & 1 & 2 & 3 \\
2 & 3 & 1 & 1 & 3 & 4 \\
2 & 4 & 0 & 2 & 4 & 6 \\
2 & 5 & 1 & 2 & & 5 & 7 \\
2 & 6 & 0 & 2 & 1 & 7 & 12 \\
2 & 7 & 1 & 2 & 1 & 8 & 13 \\
2 & 8 & 1 & 3 & 1 & 10 & 16 \\
2 & 9 & 1 & 4 & 1 & 12 & 19 \\
2 & 10 & 0 & 4 & 2 & 14 & 24 \\
2 & 11 & 0 & 5 & 2 & 16 & 27 \\
2 & 12 & 0 & 5 & 3 & 19 & 33 \\
2 & 13 & 0 & 6 & 3 & 21 & 36 \\
2 & 14 & 0 & 6 & 4 & 24 & 42 \\
2 & 15 & 1 & 7 & 4 & 27 & 46 \\
2 & 16 & 1 & 7 & 5 & 30 & 52 \\
2 & 17 & 1 & 7 & 6 & 33 & 58 \\
2 & 18 & 0 & 8 & 7 & 37 & 66 \\
2 & 19 & 1 & 9 & 7 & 40 & 70 \\
2 & 20 & 0 & 10 & 8 & 44 & 78 \\
2 & 21 & 1 & 10 & 9 & 48 & 85 \\
2 & 22 & 0 & 11 & 10 & 52 & 93 \\
2 & 23 & 1 & 11 & 11 & 56 & 100 \\
\hline
\end{tabular}

\begin{tabular}{|r|r|rrrrr|r|r|}
\hline$n$ & $d$ & $K_{0}$ & $K_{1}$ & $K_{2}$ & $K_{3}$ & $K_{4}$ & $N_{P}(Q)$ & $\nu(Q)$ \\
\hline 3 & 1 & 1 & & & & 1 & 1 \\
3 & 2 & 0 & 1 & & & 2 & 4 \\
3 & 3 & 1 & 1 & & & 3 & 5 \\
3 & 4 & 1 & 1 & 1 & & 5 & 11 \\
3 & 5 & 0 & 2 & 1 & & & 6 & 14 \\
3 & 6 & 0 & 3 & 0 & 1 & 9 & 24 \\
3 & 7 & 0 & 3 & 1 & 1 & 11 & 30 \\
3 & 8 & 1 & 3 & 1 & 2 & 15 & 43 \\
3 & 9 & 0 & 4 & 2 & 2 & & 18 & 52 \\
3 & 10 & 0 & 5 & 2 & 3 & & 23 & 68 \\
3 & 11 & 1 & 5 & 2 & 4 & & 27 & 81 \\
3 & 12 & 1 & 5 & 2 & 5 & 1 & 34 & 117 \\
3 & 13 & 1 & 6 & 2 & 6 & 1 & 39 & 133 \\
3 & 14 & 1 & 6 & 3 & 8 & 1 & 47 & 163 \\
3 & 15 & 0 & 7 & 3 & 10 & 1 & 54 & 190 \\
3 & 16 & 1 & 7 & 4 & 11 & 2 & 64 & 233 \\
3 & 17 & 0 & 8 & 3 & 14 & 2 & 72 & 266 \\
3 & 18 & 0 & 9 & 3 & 16 & 3 & 84 & 318 \\
3 & 19 & 1 & 9 & 3 & 19 & 3 & 94 & 355 \\
3 & 20 & 1 & 9 & 5 & 21 & 4 & 108 & 415 \\
3 & 21 & 0 & 10 & 4 & 24 & 5 & 120 & 472 \\
3 & 22 & 1 & 10 & 5 & 27 & 6 & 136 & 539 \\
3 & 23 & 0 & 11 & 5 & 30 & 7 & 150 & 602 \\
\hline
\end{tabular}

(4.6) and $K_{0} \leq 1$. A standard implementation of the branch and bound method with a linear programming code is used to determine the minimal number of nodes. At this stage we obtain at least one optimal $d$-consistent structure as solution of the integer programming problem, but not necessarily all possible ones. On the other hand, we note that when $\operatorname{dim} \mathscr{P}_{d}^{*}(J)=0$, then the corresponding constraint in the programming problem is always feasible and can be removed. For example, for $n=8$ the number of consistency conditions is 573, but only when $d \geq 72$ are all of them effective. When $d=10$, for instance, only 83 consistency conditions have a right-hand side different from zero.

Table 3 contains the results obtained by the method described above. We give, for a wide range of values of $n$ and $d$, the number of nodes of an optimal $d$-consistent structure. This number is of course a possibly unattainable lower bound on the number of nodes required by an invariant quadrature rule with degree of precision $d$ for the $n$-dimensional simplex $T_{n}$.

Once we know the minimal number of nodes, say $\nu_{L}(n, d)$, it is not difficult to obtain all the optimal and quasi-optimal $d$-consistent structures for each value of $n$ and $d$. For this, we use a combinatorial method for generating all the possible rule structures that have a fixed number of nodes $\nu_{L}(n, d)+k$, for $k=0,1,2$, etc. Then, we check the consistency conditions (4.6) and reject those rule structures which result not to be $d$-consistent. We found all the optimal $d$-consistent structures to be unique. 
TABLE 5. Optimal consistent structures for $T_{4}$

\begin{tabular}{|r|r|rrrrrrr|r|r|}
\hline$n$ & $d$ & $K_{0}$ & $K_{1}$ & $K_{2}$ & $K_{3}$ & $K_{4}$ & $K_{5}$ & $K_{6}$ & $N_{P}(Q)$ & $\nu(Q)$ \\
\hline 4 & 1 & 1 & & & & & & 1 & 1 \\
4 & 2 & 0 & 1 & & & & & 2 & 5 \\
4 & 3 & 1 & 1 & & & & & 3 & 6 \\
4 & 4 & 1 & 1 & 1 & & & & 5 & 16 \\
4 & 5 & 1 & 2 & 1 & & & & 7 & 21 \\
4 & 6 & 1 & 2 & 1 & 1 & & & 10 & 41 \\
4 & 7 & 0 & 3 & 2 & 1 & & & 13 & 55 \\
4 & 8 & 0 & 4 & 2 & 1 & 1 & & 18 & 90 \\
4 & 9 & 0 & 4 & 3 & 2 & 1 & & 23 & 120 \\
4 & 10 & 1 & 4 & 3 & 3 & 2 & & 30 & 171 \\
4 & 11 & 1 & 5 & 4 & 4 & 2 & & 37 & 206 \\
4 & 12 & 1 & 5 & 4 & 6 & 2 & 1 & 47 & 306 \\
4 & 13 & 1 & 6 & 5 & 6 & 4 & 1 & 57 & 381 \\
4 & 14 & 0 & 7 & 5 & 8 & 6 & 1 & 70 & 485 \\
4 & 15 & 1 & 7 & 5 & 10 & 7 & 2 & 84 & 616 \\
4 & 16 & 1 & 7 & 7 & 12 & 8 & 3 & 101 & 766 \\
4 & 17 & 1 & 8 & 7 & 14 & 10 & 4 & 119 & 931 \\
4 & 18 & 1 & 8 & 8 & 16 & 12 & 6 & 141 & 1161 \\
4 & 19 & 1 & 9 & 7 & 19 & 14 & 8 & 164 & 1396 \\
4 & 20 & 0 & 10 & 8 & 21 & 16 & 10 & 1 \\
4 & 21 & 0 & 10 & 9 & 24 & 18 & 13 & 1 & 221 & 1750 \\
4 & 22 & 1 & 10 & 9 & 27 & 22 & 16 & 1 & 255 & 2421 \\
4 & 23 & 1 & 11 & 10 & 30 & 23 & 21 & 1 & 291 & 2826 \\
\hline
\end{tabular}

The unique optimal $d$-consistent structures, for the same range of values of $n$ and $d$ as in Table 3, are presented in Tables 4, 5, 6 and 7. In each table, the column corresponding to $K_{i}$ has been suppressed if it contains only zero entries. On the other hand, extensive listings of quasi-optimal structures are given by the authors in [17].

An important remark is that the hypothesis that the simplex $T_{n}$ is centered at the origin is relevant only for the theoretical results (Theorems 2 and 3 ) that support the method we have used for computing the right-hand sides of the consistency conditions (4.6). However, the consistent rule structures and other numerical results given in this paper are valid for a general (nondegenerate) simplex in the $n$-dimensional Euclidean space $\mathbb{R}^{n}$. 
TABLE 6. Optimal consistent structures for $T_{5}$ and $T_{6}$

\begin{tabular}{|r|r|rrrrrrrrr|r|r|}
\hline$n$ & $d$ & $K_{0}$ & $K_{1}$ & $K_{2}$ & $K_{3}$ & $K_{4}$ & $K_{5}$ & $K_{6}$ & $K_{7}$ & $K_{8}$ & $N_{P}(Q)$ & $\nu(Q)$ \\
\hline 5 & 1 & 1 & & & & & & & & 1 & 1 \\
5 & 2 & 0 & 1 & & & & & & & 2 & 6 \\
5 & 3 & 1 & 1 & & & & & & & 3 & 7 \\
5 & 4 & 1 & 1 & 1 & & & & & & 5 & 22 \\
5 & 5 & 1 & 2 & 1 & & & & & & 7 & 28 \\
5 & 6 & 0 & 3 & 0 & 1 & 1 & & & & 11 & 68 \\
5 & 7 & 1 & 3 & 1 & 1 & 1 & & & & 14 & 84 \\
5 & 8 & 0 & 4 & 2 & 1 & 1 & 1 & & & 20 & 164 \\
5 & 9 & 1 & 4 & 3 & 1 & 2 & 1 & & & 26 & 210 \\
5 & 10 & 0 & 5 & 3 & 2 & 3 & 2 & & & 35 & 325 \\
5 & 11 & 0 & 5 & 4 & 1 & 4 & 4 & & & 44 & 470 \\
5 & 12 & 0 & 6 & 4 & 2 & 5 & 4 & 1 & 1 & 58 & 736 \\
5 & 13 & 1 & 6 & 4 & 2 & 7 & 6 & 1 & 1 & 71 & 917 \\
5 & 14 & 0 & 7 & 4 & 3 & 9 & 9 & 0 & 1 & 1 & 90 & 1272 \\
5 & 15 & 0 & 7 & 6 & 3 & 10 & 11 & 1 & 2 & 1 & 110 & 1662 \\
5 & 16 & 1 & 7 & 7 & 3 & 12 & 14 & 1 & 3 & 2 & 136 & 2218 \\
5 & 17 & 1 & 8 & 7 & 3 & 14 & 18 & 2 & 4 & 2 & 163 & 2734 \\
5 & 18 & 1 & 8 & 8 & 3 & 16 & 22 & 2 & 6 & 4 & 199 & 3649 \\
\hline
\end{tabular}

\begin{tabular}{|c|c|c|c|c|c|c|c|c|c|c|c|}
\hline \begin{tabular}{|l|l|}
$n$ & $d$ \\
\end{tabular} & $K_{0} K$ & $K_{1} K$ & $2 K$ & $3 K$ & $K_{4} K$ & 65 & $K_{6} K$ & ${ }_{7} K$ & $K_{8} \quad K_{9}$ & $N_{P}(Q$ & $\nu(Q)$ \\
\hline \begin{tabular}{|l|l}
6 & 1
\end{tabular} & 1 & & & & & & & & & & 1 \\
\hline \begin{tabular}{l|l|}
6 & 2
\end{tabular} & 0 & 1 & & & & & & & & & \\
\hline \begin{tabular}{l|l|}
6 & 3
\end{tabular} & 1 & 1 & & & & & & & & & 8 \\
\hline \begin{tabular}{l|l}
6 & 4
\end{tabular} & 1 & 1 & 1 & & & & & & & & 29 \\
\hline \begin{tabular}{l|l|}
6 & 5
\end{tabular} & 1 & 2 & 1 & & & & & & & & 36 \\
\hline \begin{tabular}{l|l|}
6 & 6
\end{tabular} & 0 & 3 & 0 & 1 & 1 & & & & & 1 & 98 \\
\hline \begin{tabular}{l|l|}
6 & 7
\end{tabular} & 0 & 3 & 2 & 1 & 1 & & & & & & 140 \\
\hline \begin{tabular}{l|l|}
6 & 8
\end{tabular} & 1 & 3 & 3 & 1 & 1 & 1 & & & & 2 & 267 \\
\hline \begin{tabular}{ll|l}
6 & 9
\end{tabular} & 1 & 4 & 3 & 2 & 2 & 1 & & & & & 351 \\
\hline 610 & 0 & 5 & 3 & 2 & 3 & 2 & 1 & & & & 644 \\
\hline $6 \mid 11$ & 1 & 5 & 4 & 3 & 4 & 3 & 1 & & & & 848 \\
\hline 612 & 0 & 6 & 4 & 4 & 5 & 4 & 1 & 1 & 1 & & 1456 \\
\hline $6 \mid 13$ & 0 & 6 & 5 & 4 & 7 & 6 & 2 & 1 & 1 & & 1911 \\
\hline $6 \mid 14$ & 0 & 7 & 5 & 5 & 8 & 9 & 3 & 1 & 1 & 10 & 2870 \\
\hline $6|15|$ & 51 & 7 & 6 & & & 12 & 4 & 2 & 21 & 13 & 3816 \\
\hline
\end{tabular}


TABLE 7. Optimal consistent structures for $T_{7}$ and $T_{8}$

\begin{tabular}{|c|c|c|c|c|c|c|c|c|c|c|c|c|}
\hline \begin{tabular}{l|l}
$n$ & $d$ \\
\end{tabular} & $K_{0}$ & $x_{1}$ & 2 & $K_{3}$ & $K_{4}$ & $K_{5}$ & $K_{6}$ & $7 K$ & 81 & $9 K$ & $N_{P}(Q)$ & $\nu(Q)$ \\
\hline 7 & 1 & & & & & & & & & & & \\
\hline 72 & 0 & 1 & & & & & & & & & 2 & \\
\hline $7 \mid 3$ & 1 & 1 & & & & & & & & & 3 & 9 \\
\hline 74 & 1 & 1 & 1 & & & & & & & & 5 & 37 \\
\hline 75 & 1 & 2 & 1 & & & & & & & & 7 & 45 \\
\hline 76 & 0 & 3 & 0 & 1 & 0 & 1 & & & & & 11 & 136 \\
\hline $7 \mid 7$ & 0 & 3 & 2 & 1 & 0 & 1 & & & & & 15 & 192 \\
\hline $\begin{array}{ll}7 & 8\end{array}$ & 1 & 3 & 2 & 0 & 1 & 2 & 1 & & & & 22 & 431 \\
\hline 79 & 0 & 4 & 3 & 2 & 1 & 2 & 1 & & & & 29 & 578 \\
\hline $7 \mid 10$ & 0 & 5 & 3 & 3 & 0 & 3 & 2 & 1 & & & 40 & 1076 \\
\hline \begin{tabular}{l|l|l}
7 & 11
\end{tabular} & 1 & 5 & 4 & 2 & 1 & 4 & 4 & 1 & & & & 1511 \\
\hline $7 \mid 12$ & 1 & 5 & 4 & 3 & 1 & 6 & 4 & 2 & 1 & 0 & 70 & 2715 \\
\hline $7 \mid 13$ & 1 & 6 & 4 & 3 & 2 & 7 & 7 & 3 & 1 & 0 & 89 & 3633 \\
\hline
\end{tabular}

\begin{tabular}{|c|c|c|c|c|c|c|c|c|c|}
\hline \begin{tabular}{l|l}
$n$ & $d$
\end{tabular} & $K_{0}$ & 1 & $K_{2}$ & 3 & $K_{4}$ & $k_{5} 1$ & $K_{6} K_{7}$ & $N_{P}(Q)$ & $\nu(Q)$ \\
\hline \begin{tabular}{l|l}
8 & 1
\end{tabular} & 1 & & & & & & & & \\
\hline $\begin{array}{ll}8 & 2\end{array}$ & 0 & 1 & & & & & & 2 & 9 \\
\hline \begin{tabular}{l|l}
8 & 3
\end{tabular} & 1 & 1 & & & & & & 3 & 10 \\
\hline \begin{tabular}{l|l}
8 & 4
\end{tabular} & 1 & 1 & 1 & & & & & 5 & 46 \\
\hline 85 & 1 & 2 & 1 & & & & & 7 & 55 \\
\hline \begin{tabular}{l|l}
8 & 6
\end{tabular} & 0 & 3 & 0 & 1 & 0 & 1 & & 11 & 183 \\
\hline $\begin{array}{ll}8 & 7\end{array}$ & 0 & 3 & 2 & 1 & 0 & 1 & & 15 & 255 \\
\hline \begin{tabular}{l|l|l|}
8 & 8
\end{tabular} & 1 & 3 & 2 & 0 & 1 & 2 & 1 & 22 & 622 \\
\hline \begin{tabular}{l|l|}
8 & 9
\end{tabular} & 1 & 4 & 3 & 2 & 1 & 2 & 1 & 30 & 835 \\
\hline 810 & 1 & 4 & 4 & 2 & 1 & 3 & 2 & 41 & 1699 \\
\hline
\end{tabular}

\section{Previously PUblished Structures Which ARE INCORReCT}

Our investigation has uncovered some errors in a list of structures in Keast, $[12$, pp. 345 and 346]. We found that at least fourteen rule structures of degrees 6, 7 and 8 listed there do not satisfy our consistency conditions (4.6). These are:

- The first four rule structures of degree 6 .

- The first six rule structures of degree 7.

- The first four rule structures of degree 8.

Dr. Keast subsequently reexamined some of his calculations (carried out in 1980). We have been informed that he has found at least one computational error which accounts for these particular errors. 


\section{BiBLIOGRAPHY}

1. M. Beckers and A. Haegemans, The construction of cubature formulas for the tetrahedron, Tech.Report TW 128, Katholieke Universiteit Leuven, Dept. of Comp. Sci., Celestijnenlaan 200A - B 3001, Leuven, Belgium, 1990.

2. H. E. Bez, A unified treatment of invariance for curve and surface defining algorithms, IMA J. Appl. Math. 30 (1983), 325-343.

3. R. Cools and A. Haegemans, Construction of fully symmetric cubature formulae of degree $4 k-3$ for fully symmetric planar regions, J. Comput. Appl. Math. 17 (1987), 173-180.

4. R. Cools and P. Rabinowitz, Monomial cubature rules since Stroud: a compilation, J. Comput. Appl. Math. 48 (1993), 309-326.

5. D. A. Dunavant, High degree efficient symmetrical Gaussian quadrature rules for the triangle, Internat. J. Numer. Methods Engrg. 21 (1985), 1129-1148.

6. T. O. Espelid, On the construction of good fully symmetric integration rules, SIAM J. Numer. Anal. 24 (1987), 855-881.

7. K. Gaterman, The construction of symmetric cubature formulas for the square and the triangle, Computing 40 (1988), 229-240.

8. _ Gruppentheoretische Konstruktion von symmetrischen Kubaturformeln, Tech. Report TR 90-1, Konrad-Zuse-Zentrum fur Informationstechnik, Berlin, 1990.

9. L Linear representations of finite groups and the ideal theoretical construction of $G$ invariant cubature formulas, Numerical Integration (T. O. Espelid and A. Genz, eds.), Kluwer, Dordrecht, 1992, pp. 25-35.

10. A. Grundmann and M. Möller, Invariant integration formulas for the $n$-simplex by combinatorial methods, SIAM J. Numer. Anal. 15 (1978), 282-290.

11. P. Keast, On the null spaces of fully symmetric basic rules, Tech. Report 134, University of Toronto, Dept. of Comp. Sci., Toronto, M5S 1A7, 1979.

12. $ـ$, Moderate degree tetrahedral quadrature formulas, Comput. Methods Appl. Mech. Engrg. 55 (1986), 339-348.

13. P. Keast and J. C. Diaz, Fully symmetric integration formulas for the surface of the sphere, SIAM J. Numer. Anal. 20 (1983), 406-419.

14. K. Keast and J. N. Lyness, On the structure of fully symmetric multidimensional quadrature rules, SIAM J. Numer. Anal. 16 (1979), 11-29.

15. J. N. Lyness and D. Jespersen, Moderate degree symmetric quadrature rules for the triangle, J. Inst. Math. Appl. 15 (1975), 19-32.

16. J. I. Maeztu, On symmetric cubature formulae for planar regions, IMA J. Numer. Anal. 9 (1989), 167-183.

17. J. I. Maeztu and E. Sainz de la Maza, Quasi-optimal consistent structures of invariant quadrature rules for the n-simplex, Tech. Report NA 94-7, Universidad del Pais Vasco, Dept. of Appl. Math. and Stat., Apdo. 644, 48080-Bilbao, Spain, 1994.

18. F. Mantel and P. Rabinowitz, The application of integer programming to the computation of fully symmetric integration formulas in two and three dimensions, SIAM J. Numer. Anal. 14 (1977), 391-425.

19. P. Rabinowitz and N. Richter, Perfectly symmetric two-dimensional integration formulas with minimal number of points, Math. Comp. 23 (1969), 765-799.

20. A. H. Stroud, Approximate calculation of multiple integrals, Prentice-Hall, Englewood Cliffs, NJ, 1971.

Departamento de Matemática Aplicada y Estadística, Universidad del País Vasco, Apartado 644, 48080-Bilbao, Spain

E-mail address: mepsaese@lg.ehu.es 